Ensayo Los textos publicados son responsabilidad exclusiva de sus autores

\title{
La innovación de las bibliotecas
}

\section{Library innovation}

\author{
Roxanda Uriarte Morataya \\ Universidad de San Carlos de Guatemala \\ roxandauriarte@gmail.com \\ https://orcid.org/0000-0003-2246-8133)
}

\section{Referencia del artículo}

Uriarte-Morataya, R. (2021). La innovación de las bibliotecas. Revista Guatemalteca de Cultura. 1(1), 36-41. DOI: 10.46954/revistaguatecultura.v1i1.8

Recibido 15/09/ 2020

Aceptado 25/01/2021

\section{Resumen}

El presente ensayo se basa en la evolución de las bibliotecas que constantemente innovan los servicios y colecciones físicas al formato digital, con la aparición de las nuevas tecnologías, con ellas los programas y plataformas informáticas para el desarrollo de las comunicaciones. En la vida cotidiana las personas generan vínculos que transmite conocimientos, lo que generó la necesidad de transmitirla, preservarla y conservar la biblioteca, es por ello que, las bibliotecas poseen un papel vital como preservadora, conservadora y comunicadora para la comunidad donde se encuentre, con el paso del tiempo han surgido nuevas necesidades por lo que han evolucionado para poder sostener los servicios, las colecciones, mobiliario y equipo para la disposición a los usuarios. 
4 Palabras clave: bibliotecas, gestión de bibliotecas, tecnologías de la información, big data, innovación, bibliotecología, brecha social, servicios bibliotecarios.

\section{Abstract}

This essay is based on the evolution of libraries, constantly innovating services and physical collections to digital format, with the appearance of new technologies, with them computer programs and platforms for the development of communications. Everyday life people generate links that transmit knowledge, which generated the need to transmit it, preserve it and conserve the library, that is why. Libraries play a vital role as preserver, conservator and communicator for the community where it is located, with the passage of time new needs have arisen so they have evolved to be able to sustain the services, collections, furniture and equipment for the provision to the public. users.

Keywords: libraries, management of libraries, information technology, big data, innovation, librarianship, social gap, library services

\section{Introducción}

El conocimiento se adquiere como contenido intelectual mediante la observación y el razonamiento; año con año nos hemos visto en la necesidad de transmitir el conocimiento y conservarlo, en este sentido las bibliotecas poseen un papel muy importante como función preservadora y de diseminación del conocimiento.

En el siguiente ensayo expresamos la forma como las bibliotecas han evolucionado a través del uso de las nuevas tecnologías, el desarrollo de las comunicaciones, asimismo la digitalización de documentos para su utilización en la virtualidad.

\section{Contenido}

Desde la tablilla de los sumerios hasta el proceso de impresión con la imprenta de Gutenberg en 1945; la Biblioteca Pública la describe Buonocore (1976) como la colección de libros, numerosa, selecta, con procesos técnicos de acuerdo al sistema adoptado para la dirección de la Biblioteca la cual presta sus servicios a la comunidad en donde se encuentre ubicada, sin ninguna clase de discriminación a los usuarios. En los años sesenta la tecnología se entendía a través por medio de procesos mecánicos, que ayudaron a las Bibliotecas a transformar sus procesos como la catalogación, clasificación y servicios; con ello la biblioteca tradicional paso a convertirse en biblioteca automatizada. 
A partir de los años noventa, las tecnologías conformaron un modelo tecnológico entre la sociedad y los servicios que brinda la biblioteca automatizada, por ejemplo contaba con servicios remotos: por vía telefónica, fax y correo electrónico; a través de internet, los catálogos OPAC, fueron vistos a través de un ordenador, lo que aperturó un nuevo servicio de consultas rápidas sobre el acervo de las colecciones de la biblioteca.

En el siglo $X X$, surgieron las bibliotecas híbridas, en este siglo, la tecnología ha sido la actora principal del desarrollo de procesos en las bibliotecas, pues la evolución de la "biblioteca automatizada" paso a ser una biblioteca capaz, no sólo de prestar el servicio en sus instalaciones físicas, sino también a nivel remoto, es decir, empezó a contar con recursos de información en formato digital. La Biblioteca hibrida se define como la biblioteca que armoniza con la tecnología y este proceso se convierte en una entidad individual de la biblioteca que la definirá a través de sus nuevos productos como lo expresa (Amat, 1990).

A través de los años, las bibliotecas han sufrido cambios, basada en las tecnologías de la información y de la participación (GarcíaGómez, 2013), esto se define como "La innovación de las bibliotecas", ya que apreciamos un verdadero cambio social de la tecnología con la biblioteca, donde se enlaza lo tradicional con lo tecnológico, tanto con recursos físicos como digitales. La innovación y las facilidades de comunicación que brinda la tecnología no terminan en la biblioteca híbrida.
Las bibliotecas cibertecas, es un concepto que está asociado al ciberespacio de una sociedad, en ella están incluidas todas las vertientes de la dimensión espacial de las redes de la comunicación que existen; este término se define a una sublevación de tiempo y espacio de la sociedad actual, está asociada a la categorización virtual ya que define expresiones tales como: cibercultura, ciberdocumento, cibertecario.

Ahora proseguimos a mencionar la modalidad de poder llegar a más usuarios que por diferentes brechas sociales, digitales $y$ participación las diversas concepciones geográficas, económicas, de espacio, de tiempo y por las pandemias que nos acaecen hoy en día, los usuarios que no pueden hacer uso de los recursos físicos de una biblioteca, aunque sea hibrida, por las diversas limitaciones para poder visitarla, de lo que estamos hablando es de la "biblioteca virtual o digital".

En cuanto a la definición "una biblioteca virtual, digital y electrónica, es una colección de documentos digitales debidamente organizados con criterios, biblioteconómicos, siguiendo estándares para los formatos digitales, con el fin de ponerla a disposición de los usuarios como lo expresa (Bariela, Daposo y Verónica, 2007).

Una verdadera biblioteca virtual o digital utiliza un sistema de integración de bibliotecas virtuales, como los CMS (Content Management System), licencias open source, copyright, open access, apoyando la gestión de aprendizaje de los LMS (Learning Management System); 
programas que organizan los contenidos digitales de la biblioteca, mostrando al usuario, una interfaz amigable de fácil uso y consulta para obtener acceso al acervo de fuentes de información especializada según el tipo de servicios que preste la biblioteca.

La implementación de una biblioteca dentro de un ambiente digital o virtual, requiere al igual que una biblioteca tradicional, una metodología adaptada a la naturaleza del requerimiento. Las etapas básicamente corresponden con la labor habitual de cualquier biblioteca tradicional, la única diferencia entre una biblioteca tradicional y una virtual o digital, son las técnicas o métodos y herramientas que utilizan para llevar a cabo cada etapa, con servicios remotos. Existen diferentes metodologías que se han utilizado en los últimos tiempos para crear bibliotecas virtuales alrededor del mundo, siendo las etapas básicas: selección, catalogación, clasificación, indización y digitalización, según (Bariela R. et al. 2007); no cuenta con ningún recurso físico, ofrece servicios mediante su colección digital y con otras bases de datos con acceso gratuito según la especialidad, asimismo, existen bases de datos privadas que se obtiene acceso por medio de una membresía.

\section{Conclusión}

A través de los años, las bibliotecas han sufrido cambios de acuerdo a las necesidades de información de los usuarios, basada en las nuevas tecnologías de la información. La biblioteca tradicional es una institución que presta servicios de información de calidad, su papel principal es la preservación, conservación y difusión de la información. La biblioteca hibrida es una mezcla entre los recursos físicos y digitales de las bibliotecas, las nuevas tecnologías abrieron campo para que los productos, servicios y las colecciones se puedan difundir y llegar a más usuarios con la implementación de la tecnología de las comunicaciones, ofrece acceso a su acervo en formato físico y a la misma vez con base en datos en línea.

La biblioteca digital y virtual es un centro de información completamente con servicios de información remotos. Tiene su portal lo que facilita al usuario, realizar búsquedas de información digital dentro de su acervo por medio de su base de datos, la característica especial es que no cuenta con ningún recurso físico, los servicios que se van implementando son casi ilimitados, desde acceso en línea se pueden ofrecer variedad de nuevos servicios: talleres, foros, exposiciones virtuales, novedades, acceder a los recursos de otras biblioteca e ingreso a las bases de datos.

La innovación ayuda a poseer una estructura definida para el buen funcionamiento de la biblioteca, tanto física como digital, a través del tiempo, con la tecnología este proceso se ha vuelto muy útil y rápido, permitiendo que la biblioteca pueda llegar a más usuarios, no importando las brechas de ubicación y tiempo, a través del acceso en línea. 


\section{Referencias}

Alonso Arévalo, J. (2016). ¿Para qué servirá la biblioteca Pública en el futuro?

Depende de su capacidad de adaptación a los imparables cambios sociales, económicos y tecnológicos. Recuperado de https://drive. google.com/file/d/10KPAtrOO_2XC7zblb7tJaPSSye9zX5FC/view

Amat, N. (1990). La biblioteca electrónica. Madrid: Germán Sánchez Ruipérez.

Bariela, R., Daposo G.N. \& Bogado, V. S., (2007). Digitalización de documentos para su utilización en una biblioteca virtual. Recuperado de:http://sedici.unlp.edu.ar/bitstream/handle/10915/19150/ Documento_completo.pdf?sequence=1\&isAllowed=y

Buonocore, D. (1976). Diccionario de bibliotecología. Buenos Aires : Marymar.

García-Gómez, F. J. (2013). Innovación en la biblioteca pública española: dónde estamos y hacia dónde vamos. Revista general de información y documentación, 23 (1). 133-150 pp. Recuperado de: http://dx.doi. org/10.5209/rev_RGID.2013.v23.n1.41449 


\title{
Sobre la autora
}

\begin{abstract}
Roxanda Uriarte Morataya
Es Bibliotecaria General y Bibliotecóloga, actualmente cursando la Maestría en Bibliotecología y Ciencia de la Información, en la Escuela de Estudios de Postgrado de la Facultad de Humanidades de la Universidad de San Carlos de Guatemala, es socia activa de Asociación Bibliotecológica de Guatemala -ABG-, apoyó con su informe de graduación para la licenciatura, con el título creación de la colección digital en la Escuela de Bibliotecología de la Biblioteca Virtual Juan José Arévalo Bermejo de la Facultad de Humanidades de la Universidad de San Carlos de Guatemala. Actualmente labora en la Facultad de Ciencias Médicas, asimismo realiza consultorías y talleres relacionados al aprendizaje e investigación.
\end{abstract}

Este texto está protegido por una licencia Creative Commons 4.0.

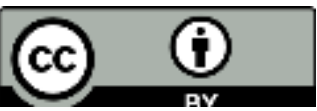

Usted es libre para compartir, copiar y redistribuir el material en cualquier medio o formato y adaptar el documento, remezclar, transformar y crear a partir del material para cualquier propósito, incluso comercialmente, siempre que cumpla la condición de atribución: usted debe reconocer el crédito de una obra de manera adecuada, proporcionar un enlace a la licencia, e indicar si se han realizado cambios. Puede hacerlo en cualquier forma razonable, pero no de forma tal que sugiera que tiene el apoyo del licenciante o lo recibe por el uso que hace. 